\title{
Twin-stage technique for occlusal rehabilitation of a mutilated dentition \\ - A case report
}

\author{
Gita Rani ${ }^{1}$ and Amarjeet Gambhir ${ }^{2 *}$ \\ ${ }^{1}$ Consultant Prosthodontist, New Delhi, India \\ ${ }^{2}$ Assistant Professor, Department of Dental \& Oral Surgery, Lady Hardinge Medical College \& \\ Hospital, New Delhi, India
}

\section{Summary}

Functional and aesthetic rehabilitation of an extremely worn dentition is one of the most challenging aspects of Prosthodontics. A thorough examination and diagnosis \& selection of appropriate occlusal scheme are key factors to achieve optimal clinical outcome. Among the variety of techniques suggested in the literature, the twin-stage technique given by Hobo \& Takayama has emerged as a popular choice for clinicians in recent times. Instead of the condylar path, it utilizes standard cuspal angle as the main determinant of articulation to produce predictable disocclusion in eccentric movements. It is relatively simple, does not require special armamentarium and can be incorporated easily with commonly used clinical techniques such as face bow transfer.

The present case report describes the full mouth rehabilitation of a severely mutilated dentition using Hobo's twin-stage technique to achieve a functionally and aesthetically favourable outcome.

\section{More Information}

${ }^{*}$ Address for Correspondence: Dr. Amarjeet Gambhir, Assistant Professor, Department of Dental \& Oral Surgery, Lady Hardinge Medical College \& Hospital, New Delhi 110001, India, Tel: +917982172082;

Email : amarjeetgambhir@gmail.com; amarjeetgambhir@yahoo.com

Submitted: 18 May 2020

Approved: 27 May 2020

Published: 28 May 2020

How to cite this article: Rani G, Gambhir A Twin-stage technique for occlusal rehabilitation of a mutilated dentition - A case report. J Oral Health Craniofac Sci. 2020; 5: 006-010.

\section{DOI: 10.29328/journal.johcs. 1001031}

Copyright: (c) 2020 Rani G, et al. This is an open access article distributed under the Creative Commons Attribution License, which permits unrestricted use, distribution, and reproduction in any medium, provided the original work is properly cited.

Keywords: Full mouth rehabilitation; Twin-stage technique; Cuspal angle; Disocclusion

(D) Check for updates

OPEN ACCESS

\section{Introduction}

Functional and aesthetic rehabilitation of an extremely worn dentition poses a distinct restorative challenge to the dentist [1]. The term occlusal rehabilitation is defined as restoration of functional integrity of dental arches by using inlays, crowns \& fixed and removable partial dentures.

Turner and Missirlian (1984) classified the patients requiring full mouth rehabilitation into three categories [2]:

- Excessive occlusal wear with loss of vertical dimension, but with space available to restore the vertical height

- Excessive wear without loss of vertical dimension of occlusion but space available

- Excessive wear without loss of vertical dimension of occlusion but with limited space

The vertical dimension of occlusion due to tooth wear is usually maintained by tooth eruption and alveolar bone growth [3]. In cases of actual loss of vertical dimension, the same needs to be increased to provide adequate space for restoration but within the limits of the accommodating musculature [4].

Over the years, a variety of occlusal schemes for full mouth rehabilitation have been proposed by various authors which includes Pankey-Man Schuyler concept [5], Hobo's twintables technique [6,7], Youdelis concept [8] \& Hobo's twinstage technique $[9,10]$. Because of its various advantages, the twin-stage technique has emerged as a popular choice among clinicians in recent times [11-14].

The present case report describes the full mouth rehabilitation of a severely mutilated dentition using Hobo's twin-stage technique to achieve a functionally and aesthetically favourable outcome.

\section{Case report}

A 30 year old male patient reported to the Department of Prosthodontics, Christian Dental College, Ludhiana with the complaints of multiple worn out teeth, poor esthetics and compromised phonetics. The patient's past medical history was not significant and there were no symptoms of temporomandibular joint dysfunction.

\section{Clinical findings}

Extraoral examination- Patient had a bilaterally symmetrical face with no abnormality in muscles of mastication and TMJ. The mandibular range of motion was within the normal limits. There was a significant difference 
of $4 \mathrm{~mm}$ between the vertical dimension of rest and occlusion (Figure 1).

Intraoral examination- Clinical examination revealed severe attrition and abrasion in relation to multiple maxillary and mandibular teeth with missing $36 \& 46$ (Figure 2). There was an anterior open bite of $3 \mathrm{~mm}$ with posterior teeth in contact in centric occlusion (Figure 3).

Specific objectives of rehabilitation in this patient included:

- Correction of anterior open bite and improvement of aesthetics

- Increase in vertical dimension of occlusion by $2 \mathrm{~mm}$

- Mutually protected occlusion anteroposteriorly with canine-guided disocclusion on lateral excursions

- Improvement in phonetics by providing adequate overbite \& overjet

Considering all these factors, full mouth rehabilitation of the dentition was planned using Hobo's twin-stage technique.

\section{Treatment procedure}

Diagnostic maxillary and mandibular impressions were made with irreversible hydrocolloid impression material (Zelgan 2002: Dust free Alginate, Dentsply DeTrey GmbH, Konstanz, Germany) and casts were obtained.

Maxillary cast was mounted on a semi-adjustable articulator (JP 30 Gnatus articulator) using a face bow transfer (JP 30 Gnatus facebow). Interocclusal records were used to articulate the mandibular cast.

Diagnostic wax up was done on the mounted casts to determine the proposed arch shape and contour, eliminate the open bite and establish the occlusion at increased vertical dimension of $2 \mathrm{~mm}$ (Figure 4).

Composite build up was done in patient's mouth keeping in view the results anticipated in the final restorations (Figure 5). The patient was kept in diagnostic and observational period of 8 weeks with weekly recalls. After 8 weeks, the patient reported satisfaction with the proposed occlusal scheme with improvement in aesthetics and speech without any significant TMJ pain or discomfort.

The actual procedure of tooth preparation for metal ceramic restorations in maxillary and mandibular arches was subsequently performed (Figure 6). Definitive impressions of the prepared maxillary \& mandibular teeth were obtained with polyvinyl siloxane impression material (Reprosil; Dentsply India, Bangalore, India) using putty wash technique. Final casts were generated from type 4 die stone (Kalrock; Kalabhai karson Pvt. Ltd, Mumbai, India) and mounted on the articulator at previously determined restored vertical dimension using face bow transfer and interocclusal records.

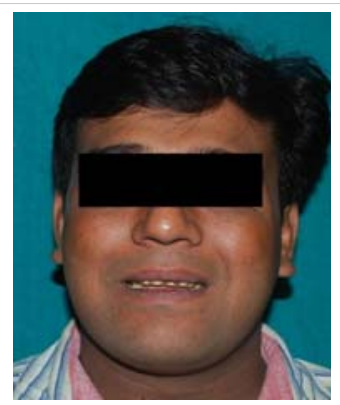

Figure 1: Preoperative extraoral view

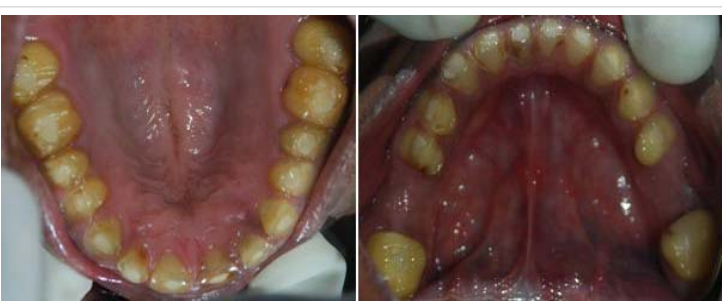

Figure 2: Maxillary and mandibular preoperative occlusal view.

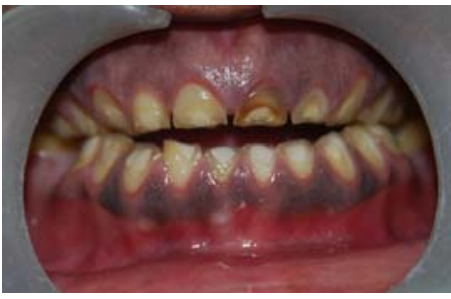

Figure 3: Intraoral frontal view in occlusion

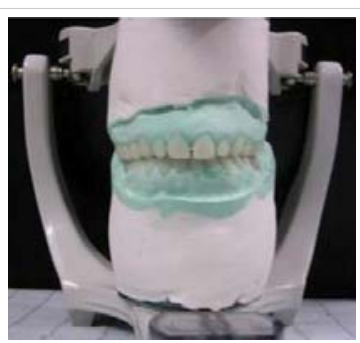

Figure 4: Diagnostic wax-up

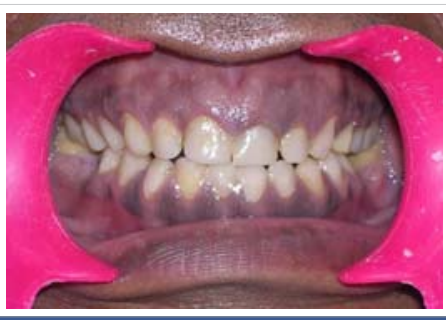

Figure 5: Composite build up at increased vertical dimension.

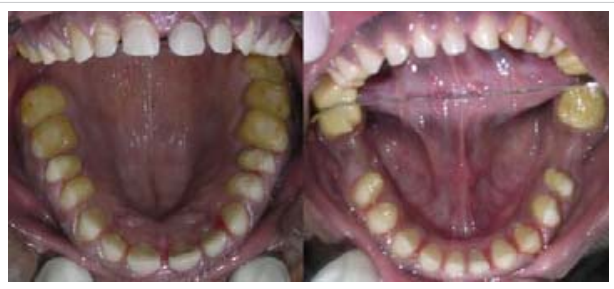

Figure 6: Maxillary and mandibulat tooth preparations. 
Tooth-colored autopolymerizing acrylic resin temporary restorations were fabricated with the help of putty index made from the diagnostic wax-up. The provisional restorations were cemented with non-eugenol zinc oxide cement and the patient was evaluated for aesthetics, comfort and speech (Figure 7).

The wax pattern for metal copings was carved and casting done. The metal copings were finished and tried in the patient's mouth (Figure 8). After satisfactory verification of the castings, they were placed on the articulator.

With the maxillary anterior segment removed, the articulator was programmed to create an effective cusp angle of $25^{0}$ posteriorly (Condition-1) for fabrication of final restorations of bilateral posterior segments.

Subsequently, the maxillary anterior segment was replaced and the articulator programmed to develop an incisal guidance of $40^{\circ}$ anteriorly (Condition-2) for fabrication of final anterior restorations.

A mutually protected (canine guided) occlusion was established which produced a standard amount of disocclusion during laterotrusive movements.

Occlusal adjustments and equilibration were carried out intraorally. Definite restorations with porcelain-fused metal crowns exhibiting a vital and natural appearance with optimal contour, shade and translucency were fabricated.

Permanent cementation was done using glass ionomer type I luting cement (Figures 9 \& 10). Disocclusion during laterotrusive movements was again confirmed in patient's mouth (Figure 11). Oral hygiene instructions were given and patient was followed up at regular intervals.

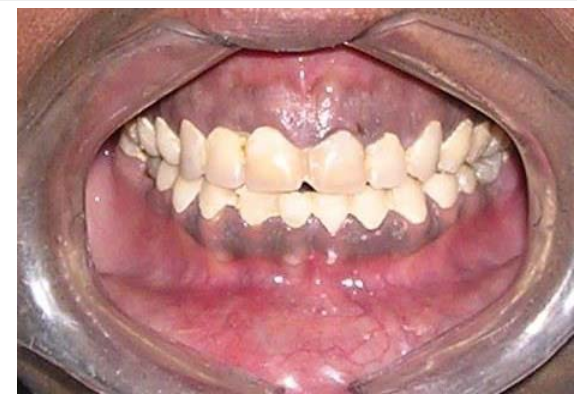

Figure 7: Provisional restorations in situ.

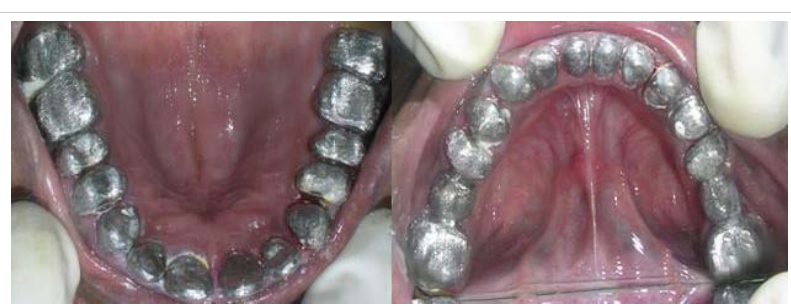

Figure 8: Try-in of metal copings.

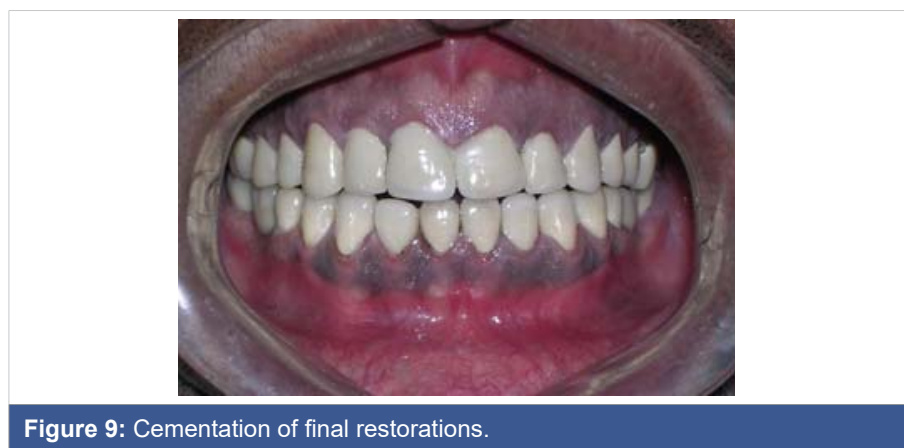

Figure 9: Cementation of final restorations.

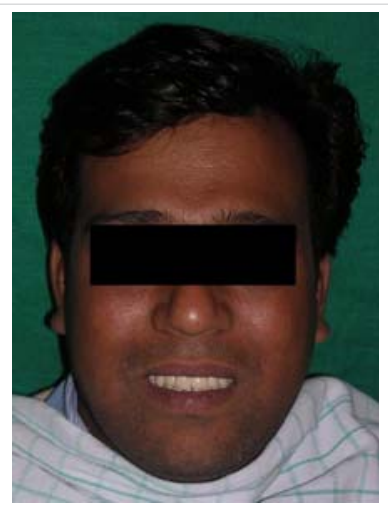

Figure 10: Postoperative extraoral view.

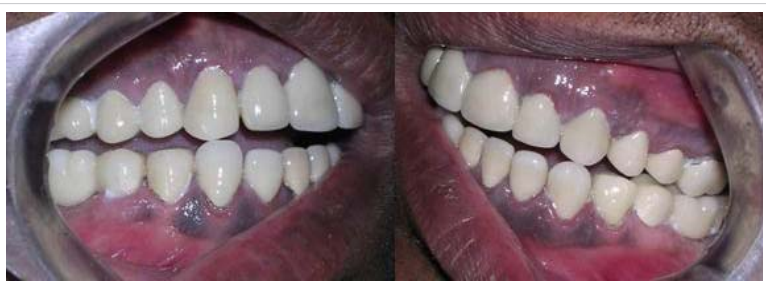

Figure 11: Canine-guided disocclusion during lateral movements.

\section{Discussion}

Full mouth rehabilitation combines the art of cosmetic dentistry with sound knowledge of the principles of gnathology. The goals of such a treatment include:

- Static coordinated occlusal contact of teeth with the condyle in comfortable reproducible position

- An anterior guidance in harmony with function in lateral eccentric position on working side

- Disocclusion of all posterior teeth in eccentric movements by anterior guidance

- Axial loading of teeth in centric relation, interproximation and function [15]

Posterior disocclusion is very important in controlling harmful lateral forces $[16,17]$. The condylar path, the incisal path and the cusp angle are the three factors which determine the amount of disocclusion during eccentric movement [18]. Initially, the condylar path was used as the main determinant of occlusion in prosthodontics. Hobo \& Takayama found that 
the condylar path is not fixed but deviates and is influenced greatly by anterior guidance $[19,20]$.

Hence, the twin-stage technique was introduced by Hobo \& Takayama as a modification of the Hobo's twin-tables technique. Instead of the condylar path, it utilizes the cuspal angle as the main determinant of articulation to produce predictable disocclusion in eccentric movements [9]. It consists of two stages:

Stage 1 (Condition 1) - Standard cusp angle is created on the articulator using predetermined adjustment values

Stage 2 (Condition 2) - Adjustment values are used to create the anterior guidance

Different adjustment values are required to reproduce standard amount of disocclusion depending upon the type of occlusal scheme chosen by the operator (mutually protected, group function or balanced occlusion) (Table 1). In mutually protected occlusion (as used in the present case), this technique helps to achieve a molar disocclusion of $1.0 \mathrm{~mm}$ in protrusion, $1.0 \mathrm{~mm}$ on non-working side and $0.5 \mathrm{~mm}$ on working side in eccentric movements for $3.0 \mathrm{~mm}$ condylar protrusion from centric relation.

Table 1: Articulator adjustment values for mutually protected articulation (degrees). Condition Condylar path

\begin{tabular}{|l|c|c|c|c|}
\hline & $\begin{array}{c}\text { Sagittal condylar path } \\
\text { inclination }\end{array}$ & $\begin{array}{c}\text { Bennett } \\
\text { angle }\end{array}$ & $\begin{array}{c}\text { Sagittal } \\
\text { inclination }\end{array}$ & $\begin{array}{c}\text { Lateral wing } \\
\text { angle }\end{array}$ \\
\hline Condition 1 & 25 & 15 & 25 & 10 \\
\hline Condition 2 & 40 & 15 & 45 & 20 \\
\hline
\end{tabular}

The twin stage procedure is a relatively simple technique and does not require sophisticated armamentarium such as fully adjustable articulator [21]. It can be applied in a variety of restorative procedures such as a single crown, fixed prosthodontics, complete dentures, implants or full mouth rehabilitation. It is specifically indicated for restorative work in patients with TMJ disorders, especially after occlusal splint therapy [22].

This procedure is contraindicated in cases with abnormal vertical inclination of posterior teeth such as those with an abnormal Curve of Spee, an abnormal Curve of Wilson and abnormally rotated \& tilted teeth [9].

\section{Conclusion}

Restoration of optimal occlusal form and function is the primary goal of full mouth rehabilitation. Proper diagnosis with meticulous planning and multidisciplinary approach to treatment are key factors for success. This case report describes the full mouth rehabilitation of a mutilated dentition using Hobo's twin-stage technique. The use of standard cusp angle as the chief determinant of occlusion produces a more predictable disocclusion during eccentric movements. It helps in avoiding detrimental forces on the teeth and supporting structures thereby restoring the optimal oral health of the patient.

\section{References}

1. Brown KE Reconstruction considerations for severe dental attrition. J Prosthet Dent. 1980; 44: 384-388.

PubMed: https://www.ncbi.nlm.nih.gov/pubmed/6997465

2. Turner KA, Missirlian DM. Restoration of the extremely worn dentition. J Prosthet Den.t 1984; 52: 467-474.

PubMed: https://www.ncbi.nlm.nih.gov/pubmed/6389829

3. Dawson PE. Functional occlusion- from TMJ to smile design. $1^{\text {st }}$ ed. New York: Elsevier Inc.; 2008.

4. Carlsson GE, Ingervall B, Kocak G. Effect of increasing vertical dimension on the masticatory system in subjects with natural teeth. $J$ Prosthet Dent. 1979; 41: 284-289.

PubMed: https://www.ncbi.nlm.nih.gov/pubmed/283228

5. Mann AW, Pankey LD. Concepts of occlusion; the P.M. philosophy of occlusal rehabilitation. Dent Clin North Am. 1963; 9: 621-636.

6. Hobo S. Twin-tables technique for occlusal rehabilitation: Part I-Mechanism of anterior guidance. J Prosthet Dent. 1991; 66: 299-303. PubMed: https://www.ncbi.nlm.nih.gov/pubmed/1800723

7. Hobo S. Twin-tables technique for occlusal rehabilitation: Part IIClinical procedures. J Prosthet Dent. 1991; 66: 471-477. PubMed: https://www.ncbi.nlm.nih.gov/pubmed/1791556

8. Schluger S, Youdelis RA, Page RC. Periodontal disease. Philadelphia: Lea \& Febiger. 1971.

9. Hobo S, Takayama H. Twin-stage procedure. Part 1: A new method to reproduce precise eccentric occlusal relations. Int J Periodont Rest Dent. 1997; 17: 113-123.

PubMed: https://www.ncbi.nlm.nih.gov/pubmed/9497706

10. Hobo S, Takayama H. Twin-stage procedure. Part 2: A clinical evaluation test. Int J Periodont Rest Dent. 1997; 17: 457-463. PubMed: https://www.ncbi.nlm.nih.gov/pubmed/9497734

11. Shetty M, Joshi N, Prasad DK, Sood S. Complete rehabilitation of a patient with occlusal wear: a case report. J Indian Prosthodont Soc. 2012; 12: 191-197.

PubMed: https://www.ncbi.nlm.nih.gov/pubmed/23997471

12. Gupta T, Banerjee A, Banerjee S, Chakraborty N, Singh R. Full mouth rehabilitation of a patient with severe attrition using Hobo twin-stage procedure. Int J Prosthodont Restor Dent. 2011; 1: 177-181. PubMed: https://www.ncbi.nlm.nih.gov/pmc/articles/PMC3341744/

13. Anil Kumar S, Radhakrishnan V, Sandhu HS, Sahoo NK. Full mouth rehabilitation of a case of rampant caries using a twin-stage procedure. Med J Armed Forces India. 2015; 71(Suppl 2): S429-434. PubMed: https://www.ncbi.nlm.nih.gov/pmc/articles/PMC4705153/

14. Verma K, Gowda EM, Kalra A, Verma R. Full mouth rehabilitation using twin-stage procedure-a case report. Ann Dent Spec. 2015; 3: 89-92.

15. Dawson PE. Evaluation, diagnosis \& treatment of occlusal problems. $2^{\text {nd }}$ ed. St Louis: Mosby. 1989; 265.

16. Tiwari B, Ladha K, Lalit A, Dwarakananda Naik B. Occlusal concepts in full mouth rehabilitation: an overview. J Indian Prosthodont Soc. 2014; 14: 344-351.

PubMed: https://www.ncbi.nlm.nih.gov/pubmed/25489156

17. Kalra A, Sandhu HS, Sahoo NK, Nandi AK, Kalra S. Full-mouth rehabilitation using twin-stage technique. Int J Oral Health Sci. 2019; 9: $40-44$.

18. Mohindra R, Kumar D, Katiyar P, Malviya A. Full mouth rehabilitation by using Hobo's twin-stage technique- a case report. University $J$ Dent Scie. 2016; 2: 105-108. 
19. Hobo S, Takayama H. Re-evaluation of the condylar path as the reference for occlusion. J Gnatho. 1995; 14: 31-40.

20. Hobo S, Takayama H. Effect of canine guidance on working condylar path. Int J Prosthodont. 1989; 2: 73-79.

PubMed: https://www.ncbi.nlm.nih.gov/pubmed/2597295
21. Choukse V, Parmar A, Sharma N, Srivastava R. Full Mouth Rehabilitation using Hobo Twin-stage Technique. Int J Prev Clin Dent Res. 2017; 4: 319-323.

22. Hobo S. Occlusion in temporomandibular disorders- treatment after occlusal splint therapy. Int Dent J. 1996; 46: 146-155.

PubMed: https://www.ncbi.nlm.nih.gov/pubmed/8886867 